\title{
Práticas educativas segundo os "Dez passos para o sucesso do aleitamento materno" em um Banco de Leite Humano
}

\author{
Educational practices in accordance with the "Ten steps \\ to successful breastfeeding" in a Human Milk Bank
}

Cristianny Miranda e Silva ${ }^{1}$

Ana Luiza Rodrigues Pellegrinelli ${ }^{1}$

Simone Cardoso Lisboa Pereira ${ }^{1}$

Ieda Ribeiro Passos ${ }^{2}$

Luana Caroline dos Santos ${ }^{1}$

${ }^{1}$ Escola de Enfermagem, Universidade Federal de Minas Gerais. Av. Professor Alfredo Balena 190/324, Santa Ifigênia. 30130-100 Belo Horizonte MG Brasil. cristiannyms@gmail ${ }^{2}$ Banco de Leite Humano da Maternidade Odete Valadares. Belo Horizonte MG Brasil.

\begin{abstract}
This article sought to evaluate educational practices in line with the "Ten Steps to Successful Breastfeeding" in a Human Milk Bank. It involved a retrospective study using sociodemographic data about the pregnancy and the baby, obtained from a nursing mothers care protocol (2009-2012). These data were associated to steps related to educational practices from the "Ten Steps." Descriptive analysis, chi-square test and Poisson regression were performed. 12,283 mothers, with a median of 29 (12-54) years old, were evaluated. The guidelines about breastfeeding received during prenatal care (step 3) prevailed among mothers aged 30-39 years and the skin to skin contact (step 4) prevailed among oriented mothers. Breastfeeding training (step 5) predominated among those who breastfed exclusively. Higher prevalence of exclusive breastfeeding (step 6), breastfeeding on demand (step 8) and use of artificial nipples (step 9) were noted among infants whose mothers were oriented. These findings indicate the important role of health professionals on mother/child training about breastfeeding, on encouragement of the skin/skin contact, exclusive breastfeeding and breastfeeding on demand. The guidelines indicated the need to improve in order to reduce the use of artificial nipples and enhance exclusive breastfeeding.
\end{abstract}

Key words Breast feeding, Milk banks, Health education, Health promotion
Resumo Este artigo objetivou avaliar práticas educativas segundo os "Dez Passos para o Sucesso do Aleitamento Materno" em Banco de Leite Humano. Estudo retrospectivo com informações sociodemográficas e gestacionais maternas e referentes ao bebê, obtidas de protocolo de atendimento de nutrizes (2009-2012). Tais dados foram associados aos passos relacionados a práticas educativas dentre os "Dez Passos". Realizou-se análise descritiva, teste qui-quadrado e regressão de Poisson. Foram avaliadas 12.283 mães, com mediana de 29 (12-54) anos de idade. As orientações recebidas sobre amamentação no pré-natal (passo 3) prevaleceram entre mães de 30-39 anos e o contato pele/pele (passo 4) entre as orientadas. O treinamento sobre amamentação (passo 5) predominou entre aquelas que amamentaram exclusivamente. Notou-se maior prevalência de amamentação exclusiva (passo 6) e sob livre demanda (passo 8) e uso de bicos artificiais (passo 9) entre os lactentes de mães orientadas. Os achados apontam importante papel do profissional da saúde no treinamento mãe/filho sobre aleitamento materno e incentivo ao contato pele/pele, amamentação exclusiva e sob livre demanda. As orientações ofertadas necessitam aprimoramento a fim de reduzir o uso de bicos artificiais e potencializar a amamentação exclusiva.

Palavras-chave Aleitamento materno, Bancos de leite, Educação em saúde, Promoção da saúde 


\section{Introdução}

A importância do aleitamento materno (AM) para a saúde materno-infantil tem sido bem documentada, sendo prioritário o desenvolvimento de estratégias de promoção desta prática. Para tal, diversos programas têm sido desenvolvidos nos últimos anos, como a Iniciativa Hospital Amigo da Criança (IHAC), a Rede Brasileira de Bancos de Leite Humano e a criação da Norma Brasileira de Comercialização de Alimentos para Lactentes $(\mathrm{NBCAL})^{1}$. Dentre esses, destacam-se aqueles de cunho educacional, pois estudos demonstram que as populações-alvo respondem satisfatoriamente às intervenções que têm como principal meta, informar as mães sobre a importância do aleitamento e orientar sobre o seu manejo ${ }^{2}$.

A Iniciativa Hospital Amigo da Criança, que visa promover, proteger e apoiar o AM, mobiliza profissionais de saúde e funcionários de hospitais e maternidades a realizarem mudanças nas políticas e rotinas desses ambientes. Tal iniciativa contempla os "Dez Passos para o Sucesso do Aleitamento Materno", os quais devem ser seguidos pelos hospitais no período pré-natal, no nascimento e após o parto ${ }^{3}$.

Os "Dez Passos para o Sucesso do Aleitamento Materno" objetivam, principalmente, treinar profissionais no sentido de informar as gestantes e nutrizes acerca dos benefícios da amamentação, bem como o manejo correto do lactente, além de fornecer informações sobre a lactação, estímulos para a produção do leite materno e resolução de dificuldades para os possíveis problemas durante a amamentação ${ }^{4}$. Uma avaliação realizada sobre a implementação desta estratégia apontou sua viabilidade de compreensão e aceitação, contudo sua efetividade pode ser aprimorada quando vinculada a uma abordagem integralizadora que inclui política, legislação, reforma do sistema de saúde e intervenções na comunidade 5 .

Os "Dez Passos" abrangem": 1- Ter uma norma escrita sobre aleitamento materno, que deve ser rotineiramente transmitida a toda equipe de saúde; 2 - Treinar toda a equipe de saúde, capacitando-a para implementar esta norma; 3Orientar todas as gestantes sobre as vantagens e o manejo do aleitamento materno; 4- Ajudar as mães a iniciar o aleitamento materno na primeira meia hora após o nascimento do bebê; 5- Mostrar as mães como amamentar e como manter a lactação, mesmo se vierem a ser separadas de seus filhos; 6- Não dar ao recém-nascido nenhum outro alimento ou bebida além do leite materno, a não ser que tal procedimento tenha uma indicação médica; 7- Praticar o Alojamento Conjunto - permitir que mãe e bebê permaneçam juntos, 24 horas por dia; 8- Encorajar o aleitamento materno sob livre demanda; 9- Não dar bicos artificiais ou chupetas a crianças amamentadas ao seio; 10- Encaminhar as mães, por ocasião da alta hospitalar, para grupos de apoio ao aleitamento materno na comunidade ou em serviços de saúde.

Nesse contexto, os Bancos de leite humano (BLH) representam polos de promoção, proteção e apoio à prática do aleitamento materno, a qual passa a ser compreendida como um processo complexo que necessita ser aprendido e apoiado pelos profissionais de saúde, mães e suas famílias ${ }^{1}$. Ademais, tais estabelecimentos objetivam orientar os familiares e incentivar o aleitamento materno por meio de estratégias para correção da pega e posição para amamentar, desde a primeira mamada; promover a continuidade da lactação; além de esclarecer de forma preventiva e corretiva sobre os problemas mamários, dentre outros ${ }^{6}$.

Assim como os bancos de leite humano, a IHAC através dos "Dez Passos para o Sucesso do Aleitamento Materno" tem se configurado como importante agente na informação e incentivo à prática da amamentação. Contudo ressaltam-se as limitações dessa estratégia no sentido de sensibilizar os profissionais de saúde em relação aos aspectos biopsicossociais que envolvem o aleitamento materno e quanto à adesão e implementação dos "Dez Passos" por parte dos dirigentes dos hospitais.

Torna-se, pois, relevante analisar a interface entre essas estratégias com a avaliação de práticas educativas, no contexto dos "Dez Passos", em um $\mathrm{BLH}$, tendo em vista a problemática que envolve o compromisso do sistema de saúde em educar adequadamente as mulheres diante da complexidade que envolve o processo da amamentação. Essa investigação poderá favorecer o planejamento de ações estratégicas, no sentido de aumentar a adesão à prática do aleitamento, em particular do "aleitamento materno exclusivo" (AME).

Assim, o presente estudo teve como objetivo avaliar práticas educativas segundo os "Dez Passos para o Sucesso do Aleitamento Materno" em um Banco de Leite Humano.

\section{Métodos}

Trata-se de estudo retrospectivo com dados secundários obtidos a partir de formulário de preenchimento obrigatório pelas mães atendidas em 
um Banco de Leite Humano de uma maternidade em Belo Horizonte (BH), com o título de Hospital Amigo da Criança. Tal título foi concedido a essa instituição, em 1999, pela Organização Mundial da Saúde (OMS), pelo Fundo das Nações Unidas para a Infância (UNICEF) e pelo Ministério da Saúde (MS).

O BLH, o qual faz parte da estrutura da referida maternidade, foi inaugurado na década de 1980 e hoje é referência no estado de Minas Gerais. Atende anualmente cerca de 3000 mães e objetiva principalmente o incentivo ao aleitamento materno e a redução da mortalidade infantil ${ }^{7}$.

Para a presente investigação, foram analisados, com enfoque nas informações relacionadas às práticas educativas no contexto dos "Dez Passos para o Sucesso do Aleitamento Materno", os formulários de atendimentos das mães que procuraram o serviço do BLH, por encaminhamento médico ou demanda espontânea, no período de 2009 a 2012.

Tais protocolos continham dados relativos aos aspectos sociodemográficos das mães (idade, escolaridade, profissão e local de residência da usuária), informações a respeito dos períodos pré e pós-gestacional (realização do pré-natal - sim/ não, e ao recebimento de orientações sobre AM nesse período - sim/não) e, sobre o recém-nascido e o parto - conduta das mães sobre AME - sim/ não, aleitamento sob livre demanda - sim/não, uso de bicos artificiais (chupetas e mamadeiras) sim/não, amamentação na sala de parto - sim/não e se houve contato pele a pele entre o recém-nascido e sua mãe logo após o nascimento - sim/não.

Ademais, foi observado, para este estudo, o treinamento mãe/filho para amamentação, dentre os motivos que levaram as mães a procurarem a equipe do BLH.

Todas as informações obtidas de tais formulários foram preenchidas manualmente pelos funcionários do $\mathrm{BLH}$, devidamente treinados para essa função, no momento de atendimento em entrevista individual com as mulheres que procuraram o serviço por motivo de intercorrências em AM ou para receberem orientações da equipe de saúde sobre dúvidas referentes a essa prática. Esse serviço atende mães de lactentes de diferentes idades, em sua maioria com dúvidas e intercorrências no início do processo da amamentação. Essas mulheres eram provenientes de diversas maternidades de Belo Horizonte, região metropolitana e outras cidades do estado.

Para avaliar os "Dez Passos" como prática educativa, as variáveis obtidas a partir do for- mulário de atendimento do referido BLH foram relacionadas com os passos 3 (informar todas as gestantes atendidas sobre as vantagens e o manejo da amamentação), 4 (ajudar a mãe a iniciar a amamentação na primeira hora após o parto), 5 (mostrar às mães como amamentar e como manter a lactação, mesmo se vierem a ser separadas de seus filhos), 6 (não dar ao recém-nascido nenhum outro alimento ou bebida além do leite materno, a não ser que tal procedimento tenha uma indicação médica), 8 (encorajar o aleitamento materno sob livre demanda) e 9 (não dar bicos artificiais - chupeta e mamadeira - a crianças amamentadas ao seio) (Figura 1).

Assim, a variável "orientação sobre aleitamento materno no pré-natal”, a qual se relaciona ao passo 3, obtida do formulário de atendimento do BLH, representou o primeiro desfecho selecionado para o presente estudo e as covariáveis, associadas a ele, foram as relativas aos dados sociodemográficos maternos (idade, escolaridade, situação funcional, local de residência - capital e proximidades) e à realização do pré-natal ( $\operatorname{sim} /$ não). Aquelas que apresentaram associação estatisticamente significante $(\mathrm{p}<0,20)$ com o desfecho no teste Qui-quadrado foram eleitas para a Regressão de Poisson, obtendo-se assim o primeiro modelo final. Essa mesma sistemática foi adotada para as demais variáveis principais elegidas do protocolo de atendimento e que tinham relação com cada um dos passos estudados (Figura 1).

Previamente à análise, realizou-se a devida verificação de consistência dos dados. Em seguida, foram calculadas as frequências e medidas de tendência central e dispersão (min-máx), além de aplicados os testes Kolmogorov-Smirnov (para verificar a adesão das variáveis à distribuição normal) e qui-quadrado de Pearson (para comparação de proporções/associações). Por fim, realizou-se Regressão de Poisson, com variância robusta, com eliminação Backward, incluindose no modelo as variáveis com valor de $\mathrm{p}<0,20$ no Qui-quadrado de Pearson. O ajuste final do modelo multivariado foi realizado por meio do Teste Hosmer e Lemeshow. O programa Statistical Package for the Social Sciences ${ }^{\circledR}$ (SPSS) versão 19.0 foi utilizado e adotou-se nível de significância de $5 \%(\mathrm{p}<0,05)$.

No tocante aos aspectos éticos, destaca-se que esse estudo faz parte do projeto "Banco de Leite Humano (BLH) de referência em Minas Gerais: caracterização, intervenção e avaliação", aprovado pelos Comitês de Ética envolvidos. 


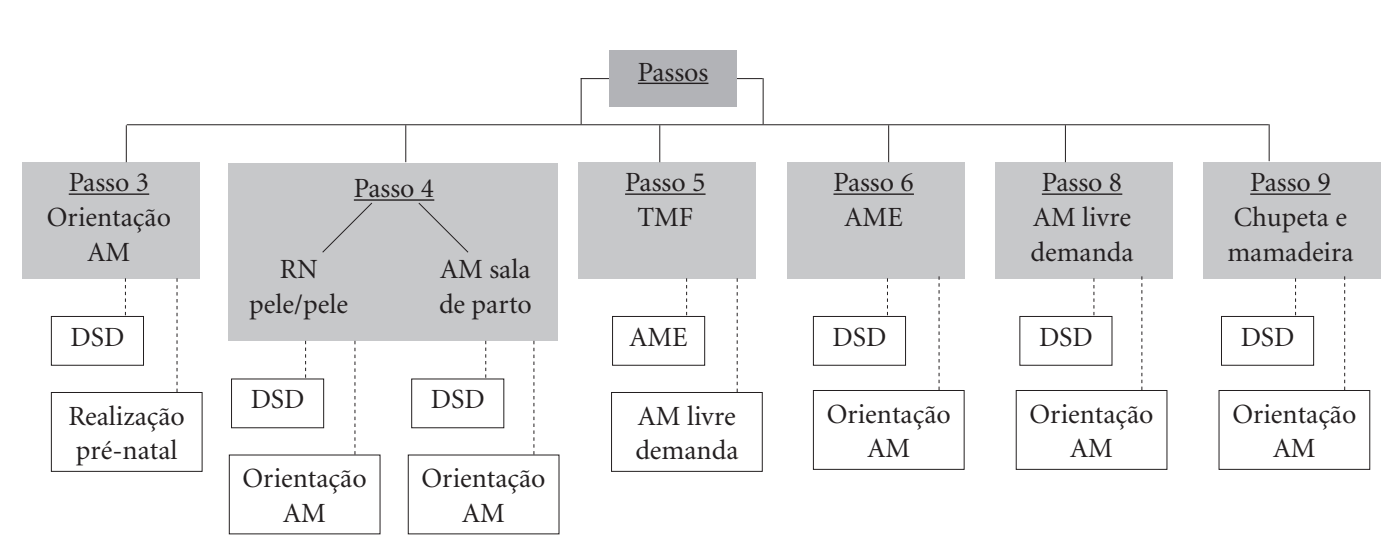

\begin{tabular}{|c|c|}
\hline & Variáveis desfecho relacionadas a cada passo estudado \\
\hline & Covariáveis relacionadas a cada variável desfecho \\
\hline AM & Aleitamento materno \\
\hline AME & Aleitamento materno exclusivo \\
\hline DSD & Dados sociodemográficos \\
\hline TMF & Treinamento mãe/filho sobre amamentação \\
\hline
\end{tabular}

Figura 1. Variáveis relacionadas aos Passos estudados e associações testadas.

\section{Resultados}

Foram avaliadas 12.283 mães no período analisado, com mediana de 29 (12 - 54) anos de idade, sendo a maioria com ensino médio. Dentre as participantes, 98,7\% referiram à realização do pré-natal - 52,4\% na rede pública (Tabela 1 ).

Com relação à variável "orientação sobre aleitamento materno no pré-natal” (Passo 3), identificou-se que apenas 38,8\% da amostra as recebeu sobre esta prática durante esse período, sendo que destas $23,9 \%$ referiram a abordagem de forma individual. Esta foi menos frequente entre as adolescentes em comparação às demais faixas etárias, e entre as analfabetas. Em contrapartida, as mulheres com profissões não remuneradas (do lar + estudante) e aquelas que residiam na cidade sede do BLH apresentaram maior frequência de orientação sobre aleitamento (Tabela 2).

A idade materna e a localização de residência permaneceram significativamente associadas à prevalência de orientação sobre aleitamento materno na análise multivariada, sendo maior entre as mulheres de 30 a 39 anos, ao contrário daquelas que residiam nas demais cidades (Tabela 3).
No que se refere ao auxilio ao início da amamentação na primeira hora após o parto (Passo 4), denota-se que a maioria das participantes informou contato pele a pele com o recém-nascido logo após o nascimento (Tabela 1). Esse contato foi mais frequente entre as que receberam orientação sobre AM no pré-natal e entre as mães adolescentes em comparação às demais faixas etárias. De forma semelhante, houve maior frequência de adolescentes que amamentaram na sala de parto.

$\mathrm{Na}$ análise multivariada, essas variáveis permaneceram associadas à prevalência do contato pele a pele, sendo esta menor entre as mulheres de 20 a 29 anos e maior entre aquelas orientadas sobre aleitamento materno. Essa prática também foi mais frequente entre as mães que amamentaram na sala de parto (Tabela 3 ).

Apesar de baixa prevalência (Tabela 1), a amamentação na sala de parto foi mais frequente entre as nutrizes que receberam orientação. A mediana de idade também diferiu conforme tal prática - 28,0 (13,0 - 49,0) anos entre aquelas que amamentaram na sala de parto e 29,0 (12,0 54,0) anos para as demais; $\mathrm{p}<0,001$ - dados não descritos em tabela. 
Tabela 1. Características sociodemográficas e do período pré e pós-gestacional de mães atendidas em um Banco de Leite Humano e dos seus recém-nascidos. Belo Horizonte/MG, 2009 - 2012.

\begin{tabular}{|c|c|c|c|}
\hline Variáveis & Categoria & $\mathbf{n}$ & $\%$ \\
\hline \multirow[t]{5}{*}{ Idade (anos) } & $<20$ & 1522 & 13,0 \\
\hline & 20 a 29 & 4780 & 41,0 \\
\hline & 30 a 39 & 4916 & 42,2 \\
\hline & 40 a 49 & 447 & 3,8 \\
\hline & $\geq 50$ & 3 & 0,0 \\
\hline \multirow[t]{5}{*}{ Escolaridade } & Analfabeto & 31 & 0,3 \\
\hline & Ensino fundamental & 2559 & 24,1 \\
\hline & Ensino Médio & 4325 & 40,7 \\
\hline & Ensino técnico & 166 & 1,6 \\
\hline & Graduação ou mais & 3541 & 33,3 \\
\hline \multirow[t]{3}{*}{ Situação funcional } & Do lar & 1927 & 19,8 \\
\hline & Estudante & 524 & 5,4 \\
\hline & Profissões remuneradas & 7257 & 74,8 \\
\hline \multirow[t]{3}{*}{ Capital estadual e proximidades } & Belo Horizonte & 7161 & 71,6 \\
\hline & Região metropolitana & 2374 & 23,8 \\
\hline & Demais cidades & 460 & 4,6 \\
\hline \multirow[t]{2}{*}{ Pré-natal } & Sim & 11649 & 98,7 \\
\hline & Não & 150 & 1,3 \\
\hline \multirow[t]{2}{*}{ Orientação sobre AM } & Sim & 3906 & 38,8 \\
\hline & Não & 6168 & 61,2 \\
\hline \multirow[t]{2}{*}{ AME } & Sim & 5804 & 59,0 \\
\hline & Não & 4025 & 41,0 \\
\hline \multirow[t]{2}{*}{ AM livre demanda } & Sim & 6664 & 70,3 \\
\hline & Não & 2811 & 29,7 \\
\hline \multirow[t]{2}{*}{ Bicos artificiais } & Sim & 2503 & 34,9 \\
\hline & Não & 4663 & 65,1 \\
\hline \multirow[t]{2}{*}{ AM sala de parto } & Sim & 2267 & 25,5 \\
\hline & Não & 6625 & 74,5 \\
\hline \multirow[t]{2}{*}{ RN pele a pele } & Sim & 6309 & 60,5 \\
\hline & Não & 4111 & 39,5 \\
\hline TMF & - & 2279 & 34,3 \\
\hline
\end{tabular}

AM: Aleitamento materno; AME: Aleitamento materno exclusivo; RN: Recém-nascido; TMF: Treinamento mãe/filho sobre amamentação.

No tocante às informações sobre o recém-nascido e o parto (passo 5), notou-se que o TMF foi mais frequente entre as mulheres que amamentaram exclusivamente $(48,4 \%$ vs $26,0 \% ; \mathrm{p}<0,001)$ e entre aquelas que amamentaram sob livre demanda $(47,3 \%$ vs $22,1 \%$; $\mathrm{p}<0,001)$ - dados não descritos em tabela. Tais evidências se mantiveram em relação à análise multivariada (Tabela 3). 
Tabela 2. Associações entre as variáveis e os passos estudados - Banco de Leite Humano, Belo Horizonte/MG, 2009 - 2012.

\begin{tabular}{|c|c|c|c|c|c|c|c|c|c|c|c|c|c|}
\hline & \multirow{3}{*}{ Variáveis } & \multirow{2}{*}{\multicolumn{2}{|c|}{$\begin{array}{c}\text { Passo } 3 \\
\begin{array}{c}\text { Orientação } \\
\text { AM }\end{array}\end{array}$}} & \multicolumn{4}{|c|}{ Passo 4} & \multirow{2}{*}{\multicolumn{2}{|c|}{$\begin{array}{c}\text { Passo } 6 \\
\text { AME }\end{array}$}} & \multirow{2}{*}{\multicolumn{2}{|c|}{$\begin{array}{c}\text { Passo } 8 \\
\text { AM livre } \\
\text { demanda }\end{array}$}} & \multirow{2}{*}{\multicolumn{2}{|c|}{$\begin{array}{c}\text { Passo } 9 \\
\text { Bicos } \\
\text { Artificiais }\end{array}$}} \\
\hline & & & & \multicolumn{2}{|c|}{ Pele a pele } & \multicolumn{2}{|c|}{$\begin{array}{c}\text { AM sala de } \\
\text { parto }\end{array}$} & & & & & & \\
\hline & & $\begin{array}{l}\text { Sim } \\
(\%)\end{array}$ & $\begin{array}{l}\text { Não } \\
(\%)\end{array}$ & $\begin{array}{l}\text { Sim } \\
(\%)\end{array}$ & $\begin{array}{l}\text { Não } \\
(\%)\end{array}$ & $\begin{array}{l}\text { Sim } \\
(\%)\end{array}$ & $\begin{array}{l}\text { Não } \\
(\%)\end{array}$ & $\begin{array}{l}\text { Sim } \\
(\%)\end{array}$ & $\begin{array}{l}\text { Não } \\
(\%)\end{array}$ & $\begin{array}{l}\text { Sim } \\
(\%)\end{array}$ & $\begin{array}{l}\text { Não } \\
(\%)\end{array}$ & $\begin{array}{l}\text { Sim } \\
(\%)\end{array}$ & $\begin{array}{l}\text { Não } \\
\text { (\%) }\end{array}$ \\
\hline \multirow[t]{7}{*}{ Idade (anos) } & $<20$ & 29,3 & 70,7 & 63,9 & 36,1 & 27,8 & 72,2 & 67,8 & 32,2 & 71,9 & 28,1 & 12,2 & 87,8 \\
\hline & 20 a 29 & 34,5 & 65,5 & 62,5 & 37,5 & 26,8 & 73,2 & 62,4 & 37,6 & 70,4 & 29,6 & 27,4 & 72,6 \\
\hline & 30 a 39 & 44,6 & 55,4 & 59,9 & 40,1 & 24,3 & 75,7 & 53,1 & 46,9 & 69,7 & 30,3 & 47,6 & 52,4 \\
\hline & 40 a 49 & 45,5 & 54,5 & 55,6 & 44,4 & 21,4 & 78,6 & 50,1 & 49,9 & 63,7 & 36,3 & 39,7 & 60,3 \\
\hline & $\geq 50$ & 0,0 & 100,0 & 50,0 & 50,0 & 0 & 100,0 & 0,0 & 100,0 & 0,0 & 100,0 & 100,0 & 0,0 \\
\hline & Analfabeto & 16,0 & 84,0 & 74,1 & 25,9 & 29,6 & 70,4 & 59,3 & 40,7 & 65,4 & 34,6 & 20,0 & 80,0 \\
\hline & Ensino fundamental & 31,3 & 68,7 & 60,9 & 39,1 & 28,6 & 71,4 & 62,5 & 37,5 & 68,3 & 31,7 & 13,7 & 86,3 \\
\hline \multirow{3}{*}{ Escolaridade } & Ensino médio & 33,1 & 66,9 & 60,1 & 39,9 & 27,5 & 72,5 & 61,2 & 38,8 & 71,7 & 28,3 & 28,4 & 71,6 \\
\hline & Ensino técnico & 38,4 & 61,6 & 58,8 & 41,2 & 25,2 & 74,8 & 51,0 & 49,0 & 72,1 & 27,9 & 44,2 & 55,8 \\
\hline & Graduação ou mais & 52,7 & 47,3 & 57,0 & 43,0 & 22,5 & 77,5 & 54,4 & 45,6 & 71,9 & 28,1 & 59,8 & 40,2 \\
\hline \multirow{3}{*}{$\begin{array}{l}\text { Profissões } \\
\text { mais relatadas }\end{array}$} & Do lar & 33,1 & 66,9 & 62,6 & 37,4 & 28,4 & 71,6 & 66,8 & 33,2 & 72,8 & 27,2 & 16,9 & 83,1 \\
\hline & Estudante & 34,2 & 65,8 & 60,1 & 39,9 & 25,9 & 74,1 & 65,5 & 34,5 & 74,4 & 25,6 & 26,2 & 73,8 \\
\hline & Profissões remuneradas & 42,5 & 57,5 & 58,7 & 41,3 & 24,4 & 75,6 & 56,1 & 43,9 & 69,6 & 30,4 & 43,5 & 56,5 \\
\hline \multirow{3}{*}{$\begin{array}{l}\text { Capital e } \\
\text { proximidades }\end{array}$} & Belo Horizonte & 41,3 & 58,7 & 58,8 & 41,2 & 25,6 & 74,4 & 59,8 & 40,2 & 73,0 & 27,0 & 37,7 & 62,3 \\
\hline & Região Metropolitana & 31,2 & 68,8 & 59,3 & 40,7 & 26,7 & 73,3 & 59,4 & 40,6 & 70,0 & 30,0 & 27,4 & 72,6 \\
\hline & Demais cidades & 31,2 & 68,8 & 41,0 & 59,0 & 13,8 & 86,2 & 36,3 & 63,7 & 40,2 & 59,8 & 18,0 & 82,0 \\
\hline \multirow[t]{2}{*}{ Pré-natal* } & Sim & 38,8 & 61,2 & 60,9 & 39,1 & 25,7 & 74,3 & - & - & - & - & - & - \\
\hline & Não & 43,5 & 56,5 & 48,5 & 51,5 & 20,7 & 79,3 & - & - & - & - & - & - \\
\hline Orientação & Sim & - & - & 64,9 & 35,1 & 27,9 & 72,1 & 59,5 & 40,5 & 73,6 & 26,4 & 41,4 & 58,6 \\
\hline $\mathrm{AM}$ & Não & - & - & 59,8 & 40,2 & 23,3 & 76,7 & 56,6 & 43,4 & 67,2 & 32,8 & 32,0 & 68,0 \\
\hline
\end{tabular}

Nota: Teste qui-quadrado. AM: Aleitamento materno; AME: Aleitamento materno exclusivo. ${ }^{\star}$ Associação não significativa $(p>0,05) \operatorname{com}$ os passos 3 e 4 (AM na sala de parto) no teste qui-quadrado.

Esse passo se associa diretamente ao Passo 6, que reforça a importância do AME, o qual foi menos frequente entre as mães com nível superior, profissões remuneradas e residentes fora da capital e região metropolitana, em contraste com a maior frequência entre as que receberam orientação sobre aleitamento materno, Tabela 2 . Ademais, as mães que declararam AME apresentaram menor mediana de idade [28 (12 - 47) vs 30 (13 - 54) anos; p < 0,001] - dados não descritos em tabela.

A prevalência de AME foi menor entre as mulheres de 30 a 39 anos, entre aquelas que cursaram até ensino fundamental completo, com profissão remunerada e as que residiam fora da capital e região metropolitana, ao contrário do observado entre as que receberam orientação sobre amamentação (Tabela 4).

O AM sob livre demanda, alvo do passo 8, foi mais frequente entre as mães adolescentes em comparação às mulheres com idade entre 40 a 49 anos, assim como o foi entre aquelas que residiam na capital e região metropolitana e as que receberam orientação sobre aleitamento (Tabela 2).

As nutrizes com profissões remuneradas e as que residiam na região metropolitana apresentaram menor prevalência de amamentação sob livre demanda, contrapondo-se àquelas que receberam orientação sobre aleitamento materno, em que essa prática foi mais frequente (Tabela 4).

Já quanto ao uso de bicos artificiais, contemplado no passo 9, pôde-se detectar que tal prática foi menor entre filhos de mães adolescentes e mais frequente entre bebês de mulheres com ensino superior, nutrizes com profissões remuneradas, residentes na capital estadual e que receberam orientação sobre aleitamento (Tabela 2).

Por fim, observou-se maior prevalência quanto à oferta de bicos artificiais aos lactentes pelas mulheres na faixa etária de 30 a 39 anos, assim como pelas estudantes. Essa prática foi menos frequente entre aquelas que residiam na região metropolitana e as orientadas sobre amamentação durante o pré-natal (Tabela 4). 
Tabela 3. Razão de prevalência entre as variáveis e os passos 3, 4 e 5 - Banco de Leite Humano, Belo Horizonte/MG, $2009-2012$.

\begin{tabular}{|c|c|c|c|c|c|c|c|c|c|}
\hline & \multirow{3}{*}{ Variáveis } & \multirow{2}{*}{\multicolumn{2}{|c|}{$\begin{array}{c}\text { Passo } 3 \\
\text { Orientação AM }\end{array}$}} & \multicolumn{4}{|c|}{ Passo 4} & \multirow{2}{*}{\multicolumn{2}{|c|}{$\begin{array}{c}\text { Passo } 5 \\
\text { TMF }\end{array}$}} \\
\hline & & & & \multicolumn{2}{|c|}{ RN pele a pele } & \multicolumn{2}{|c|}{ AM sala de parto } & & \\
\hline & & RP & IC 95\% & RP & IC 95\% & RP & IC 95\% & RP & IC 95\% \\
\hline \multirow[t]{4}{*}{ Idade (anos) } & $<20$ & 1,00 & -- & -- & -- & 1,00 & -- & -- & -- \\
\hline & 20 a 29 & 1,23 & $1,11-1,38$ & -- & -- & 0,95 & $0,84-1,07$ & -- & -- \\
\hline & 30 a 39 & 1,58 & $1,42-1,75$ & -- & -- & 0,84 & $0,74-0,95$ & -- & -- \\
\hline & $\geq 40$ & 1,54 & $1,31-1,81$ & -- & -- & 0,68 & $0,52-0,89$ & -- & -- \\
\hline \multirow{3}{*}{$\begin{array}{l}\text { Capital e } \\
\text { proximidades }\end{array}$} & Belo Horizonte & 1,00 & -- & 1,00 & -- & 1,00 & -- & -- & -- \\
\hline & Região Metropolitana & 0,79 & $0,74-0,85$ & 1,02 & $0,97-1,07$ & 1,06 & $0,96-1,16$ & -- & -- \\
\hline & Demais cidades & 0,78 & $0,67-0,91$ & 0,73 & $0,62-0,85$ & 0,56 & $0,42-0,76$ & -- & -- \\
\hline \multirow{2}{*}{$\begin{array}{l}\text { Orientação } \\
\text { AM }\end{array}$} & Não & -- & -- & 1,00 & -- & 1,00 & -- & -- & -- \\
\hline & $\operatorname{Sim}$ & -- & -- & 1,09 & $1,06-1,13$ & 1,22 & $1,13-1,32$ & -- & -- \\
\hline \multirow{3}{*}{$\begin{array}{l}\text { Profissões } \\
\text { mais } \\
\text { relatadas }\end{array}$} & Do lar & & & 1,00 & -- & & & & \\
\hline & Estudante & -- & -- & 0,94 & $0,85-1,05$ & -- & -- & -- & -- \\
\hline & Profissões remuneradas & -- & -- & 0,91 & $0,87-0,96$ & -- & -- & -- & -- \\
\hline \multirow[t]{2}{*}{ AME } & Não & -- & -- & -- & -- & -- & -- & 1,00 & -- \\
\hline & Sim & -- & -- & -- & -- & -- & -- & 1,45 & $1,31-1,61$ \\
\hline \multirow{2}{*}{$\begin{array}{l}\text { AM livre } \\
\text { demanda }\end{array}$} & Não & -- & -- & -- & -- & -- & -- & 1,00 & -- \\
\hline & $\operatorname{Sim}$ & -- & -- & -- & -- & -- & -- & 1,62 & $1,43-1,83$ \\
\hline
\end{tabular}

AM: Aleitamento materno; AME: Aleitamento materno exclusivo; BLH: Banco de Leite Humano; IC: Intervalo de confiança; RN: Recém-nascido; TMF: treinamento mãe e filho sobre amamentação; RP: Razão de prevalência.

Tabela 4. Razão de prevalência entre as variáveis e os passos 6, 8 e 9 - Banco de Leite Humano, Belo Horizonte/MG, 2009 - 2012.

\begin{tabular}{|c|c|c|c|c|c|c|c|}
\hline & \multirow[t]{2}{*}{ Variáveis } & \multicolumn{2}{|c|}{$\begin{array}{c}\text { Passo } 6 \\
\text { AME }\end{array}$} & \multicolumn{2}{|c|}{$\begin{array}{c}\text { Passo } 8 \\
\text { AM livre demanda }\end{array}$} & \multicolumn{2}{|c|}{$\begin{array}{c}\text { Passo } 9 \\
\text { Bicos artificiais }\end{array}$} \\
\hline & & $\mathbf{R P}$ & IC 95\% & $\mathbf{R P}$ & IC 95\% & RP & IC $95 \%$ \\
\hline \multirow[t]{4}{*}{ Idade (anos) } & $<20$ & 1,00 & -- & -- & -- & 1,00 & -- \\
\hline & 20 a 29 & 0,97 & $0,90-1,05$ & -- & -- & 2,14 & $1,60-2,86$ \\
\hline & 30 a 39 & 0,81 & $0,74-0,88$ & -- & -- & 3,12 & $2,33-4,17$ \\
\hline & $\geq 40$ & 0,75 & $0,64-0,89$ & -- & -- & 2,76 & $1,98-3,84$ \\
\hline \multirow[t]{5}{*}{ Escolaridade } & Analfabeto & 1,00 & -- & -- & -- & -- & -- \\
\hline & Ensino fundamental & 0,70 & $0,54-0,92$ & -- & -- & -- & -- \\
\hline & Ensino médio & 0,70 & $0,54-0,91$ & -- & -- & -- & -- \\
\hline & Ensino técnico & 0,66 & $0,48-0,90$ & -- & -- & -- & -- \\
\hline & $\geq$ Graduação & 0,67 & $0,52-0,88$ & -- & -- & -- & -- \\
\hline \multirow[t]{3}{*}{ Ocupação } & Do lar & 1,00 & -- & 1,00 & -- & 1,00 & -- \\
\hline & Estudante & 0,91 & $0,82-1,02$ & 0,99 & $0,92-1,09$ & 2,28 & $1,74-3,00$ \\
\hline & Demais profissões remuneradas & 0,86 & $0,83-0,94$ & 0,93 & $0,89-0,97$ & 1,99 & $1,69-2,35$ \\
\hline \multirow{3}{*}{$\begin{array}{l}\text { Capital e } \\
\text { proximidades }\end{array}$} & Belo Horizonte & 1,00 & -- & 1,00 & -- & 1,00 & -- \\
\hline & Região Metropolitana & 0,96 & $0,91-1,02$ & 0,94 & $0,90-0,98$ & 0,78 & $0,71-0,87$ \\
\hline & Demais cidades & 0,59 & $0,48-0,73$ & 0,52 & $0,43-0,62$ & 0,55 & $0,40-0,74$ \\
\hline \multirow{2}{*}{$\begin{array}{l}\text { Orientação } \\
\text { AM }\end{array}$} & Não & 1,00 & -- & 1,00 & -- & 1,00 & -- \\
\hline & $\operatorname{Sim}$ & 1,08 & $1,03-1,13$ & 1,09 & $1,05-1,13$ & 1,14 & $1,06-1,23$ \\
\hline
\end{tabular}

AM: Aleitamento materno; AME: Aleitamento materno exclusivo; IC: Intervalo de confiança; RP: Razão de prevalência. 


\section{Discussão}

O aleitamento materno beneficia a mãe, a criança e a família e deve ser incentivado principalmente com orientações e estratégias educativas durante os períodos pré e pós-natal mediadas por equipe multidisciplinar de saúde. Esse processo, no entanto, deve ser entendido como a busca do diálogo e o empoderamento, de forma que a mulher adquira autonomia para a realização desta prática ${ }^{8}$.

O aconselhamento, individual ou coletivo, representa então estratégia importante para a elevação dos índices de $\mathrm{AM}^{9}$. Pôde-se notar, no presente estudo, que as orientações sobre o aleitamento materno associaram-se de forma positiva à adesão a todos os passos. Quando realizadas de forma coletiva destacam-se a troca de experiência entre gestantes, a realização de atividades práticas com intuito de abordar assuntos como pega correta, posição do bebê e manejo clínico, palestras multidisciplinares, entre outros ${ }^{2}$. Entretanto, a orientação individual se faz necessária, sobretudo entre as mães adolescentes, analfabetas e as que não moram na capital, tal como aqui evidenciado, devido à dificuldade de compreensão aplicada das informações e acesso aos serviços de saúde.

Os achados também demonstraram que as práticas do AME e sob livre demanda foram favorecidas pela realização prévia de treinamento mãe e filho entre as mulheres, corroborando ${ }^{10}$ e evidenciando a importância da orientação aplicada para oportunizar a continuidade do aleitamento materno exclusivo até os 6 meses de idade 8 .

Além das orientações, ressalta-se o papel do contato pele a pele na primeira hora de vida como contribuinte para o aumento da duração da amamentação. Constitui importante instrumento para o estabelecimento do vínculo mãefilho, apesar de ainda ser pouco explorado pelos profissionais e gestantes. Essa situação pode decorrer de desinformação em relação ao direito a amamentação na sala de parto e ao contato pele a pele, sendo o pré-natal a ocasião ideal para a difusão de tais práticas ${ }^{11}$.

Em contrapartida, tal desinformação poderia justificar a associação negativa entre o contato pele a pele e o aleitamento na sala de parto e mulheres mais velhas ou com profissões remuneradas. Nesse sentido, em estudo realizado na Tanzânia ${ }^{12}$ as mulheres mais jovens apresentaram maior risco de atraso no início da amamentação, diferente do evidenciado na presente pesquisa em que mulheres mais velhas amamentaram menos na sala de parto. Ademais, mães que precisam contribuir com seu trabalho para o sustento da família podem ter menor acesso às informações sobre $\mathrm{AM}^{13}$.

O contato pele a pele e a amamentação na sala de parto podem sofrer ainda a influência das normas e rotinas dos serviços de saúde, do investimento em capacitações e da tradição institucional $^{14}$, os quais podem caracterizar a sistemática das maternidades do interior do estado onde a Iniciativa Hospital Amigo da Criança ainda não atingiu ampla divulgação e/ou implementação.

Já com relação à importância da manutenção do aleitamento materno, que se relaciona diretamente ao aleitamento materno exclusivo e à amamentação sob livre demanda, a efetividade dos programas de apoio regular e suporte às nutrizes têm sido extensamente demonstrados. Estes visam à promoção do aleitamento materno e incremento do conhecimento das mulheres durante a gestação ${ }^{15,16}$.

$O$ resultado do presente estudo confirma o encontrado por Silva et al. ${ }^{17}$, o qual demonstra que, durante o período pré-natal, as informações transmitidas às mães sobre aleitamento materno podem não ter sido completamente assimiladas levando-as a afirmarem o desconhecimento sobre tais orientações. Além disso, segundo Campos et al. ${ }^{18}$, a falta de conhecimento pode contribuir para a introdução precoce de outros alimentos, interferindo negativamente no AME.

Nesse contexto, vários estudos têm relacionado o conhecimento em amamentação e escolaridade materna, demonstrando maior prevalência de aleitamento entre os lactentes das mães com mais anos de estudo, provavelmente pela maior valorização dessa prática por esse grupo ${ }^{19}$. No entanto, essa relação ainda é muito discutida na literatura, Bbaale ${ }^{20}$ por exemplo, demonstra que aquelas que estudaram menos amamentaram exclusivamente por menor período de tempo.

Além disso, Barbosa et al. ${ }^{21}$ afirmaram que a duração do aleitamento materno é influenciada pelo nível socioeconômico dos países, já que naqueles em desenvolvimento a duração da amamentação é menor. Webb-Girard et al. ${ }^{22}$ comentam que, entre as populações pobres, ainda persistem diferenças marcantes quanto ao acesso e qualidade da orientação alimentar e nutricional prestada pelos profissionais de saúde, em que as crianças são submetidas a regimes alimentares marcados pelo oferecimento precoce de alimentos complementares. Ademais, outros autores ressaltam a necessidade do trabalho materno fora de casa, devido à insegurança financeira, corroborando os resultados do presente artigo, o qual demonstrou maior risco de interrupção do AM sob livre demanda entre mães 
que trabalhavam fora do lar e que residiam na região metropolitana ${ }^{15,19,20}$.

O incentivo para a criação de salas de apoio à amamentação nas empresas, segundo recomendação do Ministério da Saúde ${ }^{23}$ também deve ser considerado. Ademais, demandam-se orientações às nutrizes quanto à possibilidade de manter $\mathrm{o}$ aleitamento, mesmo longe de casa, através de esclarecimentos quanto à coleta e armazenamento do leite humano ordenhado, no sentido de apoiar o aleitamento materno entre mães trabalhadoras.

Demétrio et al. ${ }^{24}$ também apontaram que a residência materna fora da capital representou fator de proteção ao aleitamento materno prolongado. Os autores justificaram tal achado em virtude da preservação da prática de vida saudável e cooperação entre os indivíduos residentes nesses locais.

Cabe destacar que a presente amostra é constituída principalmente por mulheres de escolaridade até o ensino médio e que, embora tendo profissão remunerada, podem não contar com apoio legal para amamentação, ou que ainda tenham dificuldade de locomoção até a unidade de saúde para realizarem um pré-natal adequado ou buscarem auxílio no caso de intercorrências na prática do aleitamento materno.

Com relação aos bicos artificiais os achados corroboram a associação de maior uso da chupeta com menor escolaridade materna ${ }^{25}$. Em contraste, outra pesquisa apontou que o nível de escolaridade das mães parece não interferir na oferta de chupeta aos seus filhos, tendo em vista a crença de ser algo natural e necessário para acalentar a criança ${ }^{26}$.

Ademais, diferente do evidenciado, Pizzol et al. ${ }^{27}$ denotaram em seu estudo que mães mais jovens (com idade inferior a 20 anos) foram as que mais ofereceram chupeta aos seus filhos. Segundo esses autores mães mais maduras são menos influenciadas a opiniões e sugestões de familiares quanto à saúde e ao tipo de alimentação dos seus filhos. Além disso, a necessidade de uso de chupeta e mamadeira caracterizam mitos e crenças enraizadas na sociedade e relacionadas a fatores culturais ou familiares.

Já Rocci e Fernandes ${ }^{28}$ demonstraram em sua pesquisa que, apesar de os bebês terem nascido em um Hospital Amigo da Criança, as mulheres são pouco orientadas para evitarem o uso de chupeta pelos lactentes. Os autores concluíram que isso faz parte da cultura do país e ressaltaram a importância do profissional de saúde no processo educativo.

No tocante à utilização de mamadeira, corroborando este estudo, a literatura demonstra que mulheres com menor escolaridade oferecem mais mamadeira a seus lactentes ${ }^{25}$. Ademais, Silvério et al. ${ }^{26}$ afirmam que essas mães podem oferecer esses artifícios para seus filhos, tendo em vista serem hábitos bastante difundidos e culturalmente arraigados no Brasil e em outros países.

Finalmente, as mães que residiam na região metropolitana foram as que menos ofereceram bicos artificiais aos seus filhos, provavelmente pelo fato de terem sido as que mais amamentaram sob livre demanda. Segundo Moimaz et al. ${ }^{29}$ o aleitamento materno supre as duas "fomes" do recém-nascido, incluindo a necessidade fisiológica de nutrição e a de sucção.

Apesar dos importantes resultados ora detectados, apontam-se as limitações referentes ao delineamento do estudo (sujeito à causalidade reversa), ao viés de memória das mães e a utilização de dados secundários (ausência de dados em alguns protocolos). Entretanto, destaca-se o grande número de mulheres atendidas, a relevância do tema abordado e a sua interface com o local de realização da pesquisa.

Por fim, pode-se concluir que os achados apontam para a importância do profissional da saúde no treinamento mãe/filho sobre aleitamento materno e incentivo ao contato pele/pele, amamentação exclusiva e sob livre demanda. Ressalta-se a necessidade de aprimoramento das orientações ofertadas a fim de reduzir o uso de bicos artificiais e potencializar a amamentação exclusiva. 


\section{Colaboradores}

CM Silva e ALR Pellegrinelli participaram da coleta de dados, concepção, análise estatística, análise dos resultados e redação do artigo. SCL Pereira e IR Passos participaram da revisão crítica do artigo. LC Santos participou da orientação da pesquisa, análise dos resultados, revisão crítica e aprovação da versão a ser publicada.

\section{Referências}

1. Espírito Santo LC. Formulação e implementação de políticas públicas de promoção, proteção e apoio ao aleitamento materno. In: Silva IA, organizador. PROENF: Saúde Materna e Neonatal. Programa de Atualização em Enfermagem. Porto Alegre: Artmed; 2010. p. 9-38.

2. Demitto MO, Silva TC, Páschoa ARZ, Mathias TAF, Bercini LO. Orientações sobre amamentação na assistência pré-natal: uma revisão integrativa. Rev Rene 2010; 11(n. esp.):223-229.

3. Figueredo SF, Mattar MJG, Abrão ACFV. Iniciativa Hospital Amigo da Criança - uma política de promoção, proteção e apoio ao aleitamento materno. Acta Paul Enferm 2012; 25(3):459-463.

4. Brasil. Ministério da Saúde (MS). Secretaria de Atenção à Saúde. Área Técnica de Saúde da Criança e Aleitamento Materno. Departamento de Ações Programáticas Estratégicas. Iniciativa Hospital Amigo da Criança. Brasília: MS; 2011. [acessado 2013 mar 09]. Disponível em: http://bvsms.saude.gov.br/bvs/publicacoes/iniciativa_hospital_amigo_crianca.pdf

5. United Nations Children's Fund (UNICEF) Innocenti Research Centre. Celebrating the Innocenti Declaration on the Protection, Promotion and Support of Breastfeeding: past achievements, present challenges and the way forward for infant and young child feeding. Florence: UNICEF Innocenti Research Centre; 2005. [acessado 2015 jul 25]. Disponível em: http:// www.unicef.org/nutrition/files/Innocenti_plus15_ BreastfeedingReport.pdf

6. Alves VH, Rodrigues DP, Branco MBLR, Souza MRP, Souza RRB, Medeiros FVA. Banco de leite humano na perspectiva da mulher doadora. Rev Rene 2013; 14(6):1168-1176.

7. Fundação Hospitalar do Estado de Minas Gerais (FHEMIG). Banco de notícias. Notícias de Minas. Banco de Leite MOV completa 24 anos e realiza atividades educativas, 2010. [acessado 2014 jan 23]. Disponível em: http://www.fhemig.mg.gov.br/pt/banco- de-noticias/20-noticias-de-minas/1643-banco-de-leite-movcompleta-24-anos-e-realiza-atividades-educativas-

8. Nascimento VC, Oliveira MIC, Alves VH, Silva KS. Associação entre as orientações pré-natais em aleitamento materno e a satisfação com o apoio ao amamentar. Rev Bras Saúde Matern Infant 2013; 13(2):147-159.

9. Vieira TO, Vieira GO, Oliveira NF, Mendes CMC, Giugliani ERJ, Silva LR. Duration of exclusive breastfeeding in a Brazilian population: new determinants in a cohort study. BMC Pregnancy Childbirth 2014; 14:175. 
10. Nascimento MBR, Reis MAM, Franco SC, Issler $\mathrm{H}$, Ferraro AA, Grisi SJFE. Exclusive Breastfeeding in Southern Brazil: Prevalence and Associated Factors. Breastfeed Med 2010; 5(2):79-85.

11. Barbosa V, Orlandi FS, Dupas G, Beretta MIR, Fabbro MRC. Aleitamento materno na sala de parto: a vivência da puérpera. Cienc Cuid Saúde 2010; 9(2):366-373.

12. Victor R, Baines SK, Agho KE, Dibley MJ. Determinants of breastfeeding indicators among children less than 24 months of age in Tanzania: a secondary analysis of the 2010 Tanzania Demographic and Health Survey. BMJ Open 2013; 3(1):1-8.

13. Guimarães LAOP, Marçal F, Zuffi FB, Ribeiro MC, Rodrigues LR, Fonseca-Machado MO. PET-Saúde na identificação do conhecimento de gestantes sobre aleitamento materno. Cienc Cuid Saude 2012; 11(3):454462.

14. Boccolini CS, Carvalho ML, Oliveira MIC, Vasconcellos AGG. Fatores associados à amamentação na primeira hora de vida. Rev Saude Publica 2011; 45(1):69-78.

15. Seid AM, Yesuf ME, Koye DN. Prevalence of exclusive Breastfeeding practices and associated factors among mothers in Bahir Dar city, Northwest Ethiopia: a community based cross-seccional study. Int Breastfeed J 2013; 8:14.

16. Sasaki Y, Ali M, Kakimoto K, Saroeun O, Kanal K, Kuroiwa C. Predictors of exclusive breast-feeding in early infancy: a survey report from Phnom Penh, Cambodia. J Pediatr Nurs 2010; 25(6):463-469.

17. Silva NM, Waterkemper R, Silva EF, Cordova EP, Bonilha ALL. Conhecimento de puérperas sobre amamentação exclusiva. Rev Bras Enferm 2014; 67(2):290295.

18. Campos AMS, Chaoul CO, Carmona EV, Higa R, Vale IN. Prática de aleitamento materno exclusivo informado pela mãe e oferta de líquidos aos seus filhos. Rev Latinoam Enferm 2015; 23(2):283-290.

19. Martins CC, Vieira GO, Vieira TO, Mendes CMC. Fatores de riscos maternos e de assistência ao parto para interrupção precoce do aleitamento materno exclusivo: estudo de coorte. Rev Baiana Saúde Pública 2011; 35(Supl. 1):167-178.

20. Bbaale E. Determinants of Early Initiation, Exclusiveness, and Duration of Breastfeeding in Uganda. J Health Popul Nutr 2014; 32(2):249-260.

21. Barbosa LN, Santos NC, Moraes MAM, Rizzardi SD, Corrêa EC. Prevalência de práticas educativas acerca do aleitamento materno exclusivo (AME) em Cuiabá - MT. Esc Anna Nery 2015; 19(1):147-153.
22. Webb-Girard A, Cherobon A, Mbugua S, KamauMbuthia E, Amin A, Sellen DW. Food insecurity is associated with attitudes towards exclusive breastfeeding among women in urban Kenya. Matern Child Nutr 2012; 8(2):199-214.

23. Brasil. Ministério da Saúde (MS). Agência Nacional de Vigilância Sanitária (Anvisa). Nota técnica conjunta $n^{\circ} 01 / 2010$. Sala de apoio à amamentação em empresas. Brasília: MS, Anvisa. [acessado 2014 maio 22]. Disponível em: http://bvsms.saude.gov.br/bvs/publicacoes/sala_apoio_amamentacao_empresas.pdf.

24. Demétrio F, Jesus Pinto EJ, Assis AMO. Fatores associados à interrupção precoce do aleitamento materno: um estudo de coorte de nascimento em dois municípios do Recôncavo da Bahia, Brasil. Cad Saude Publica 2012; 28(4):641-654.

25. Rigotti RR, Oliveira MIC, Boccolini CS. Associação entre o uso de mamadeira e de chupeta e a ausência de amamentação no segundo semestre de vida. Cien Saude Colet 2015; 20(4):1235-1244.

26. Silvério KCA, Ferreira APS, Johanns MC, Wolf A, Furkim AM, Marques JM. Relação de escolaridade, faixa etária e profissão de mães com a oferta de chupeta e mamadeira a seus filhos. Rev CEFAC 2012; 14(4):610615.

27. Pizzol KEDC, Boeck EM, Santos LFP, Lunardi N, Oliveira GJPL. Influência do ambiente familiar e da condição socioeconômica na introdução e na manutenção de hábito de sucção não nutritiva. Rev Odontol UNESP 2011; 40(6):296-303.

28. Rocci E, Fernandes RAQ. Dificuldades no aleitamento materno e influência no desmame precoce. Rev Bras Enferm 2014; 67(1):22-27.

29. Moimaz SAS, Rocha NB, Garbin AJI, Saliba O. Relação entre aleitamento materno e hábitos de sucção não nutritivos. Cien Saude Colet 2011; 16(5):2477-2484.

Artigo apresentado em 31/07/2015

Aprovado em 17/11/2017

Versão final apresentada em 19/11/2017 
